CLINICAL ASSOCIATIONS WITH BACTERIAL

TRANSLOCATION

O'Boyle CJ, Sagar PM, Murchan P, Mitchell CJ, MacFieJ

Combined Gastroenterology Service, Scarborough

Hospital, North Yorkshire

Gut translocation of bacteria (BT) may be important in the aetiology of multi-organ failure. Surprisingly little information is available as to the prevalence of this phenomenon in different patient groups. In this study gut translocation of bacteria was assessed by culture of mesenteric lymph nodes and serosal scrapings taken at laparotomy. Standard microbiological techniques were used.

A total of 448 patients was studied BT to mesenteric lymph nodes was identified in 69 patients (15.4\%). Comparison of individual patient groups revealed no significant differences in the incidence of translocation between benign and malignant disease ( $17 \%$ versus $13 \%$ ), inflammatory bowel disease and other GI disease ( $20 \%$ versus $15 \%$ ), significant weight loss and no weight loss (10\% versus $15 \%)$ and total parenteral nutrition and enteral feeding ( $18 \%$ versus $14 \%)$. However,

translocation was more common in patients with intestinal obstruction $(30 \%)$ than in those without obstruction $(13 \%, p<0.01)$. The more distal the level of obstruction, the greater the incidence of translocation. Postoperative septic complications were more common in patients with confirmed BT $(p<0.001)$

We conclude that the incidence of BT is highest in patients with intestinal obstruction.

F270

BACTERIAL COLONIZATION OF THE UPPER GASTROINTESTINAL TRACT IS ASSOCIATED WITH SEPTIC MORBIDITY IN SURGICAL PATIENTS

OBoyle CJ, Murchan P, Dave K, Mitchell CJ, MacFie J.

Combined Gastroenterology Unit, Scarborough General Hospital,

Scarborough, North Yorkshire.

Bacterial colonization of the normally sterile upper gastrointestinal tract may predispose to septic morbidity in surgical patients. We have attempted to confirm this in a prospective study in which changes in proximal gut flora and septic complications have been recorded.

All patients underwent nasogastric intubation as part of standard c̊llnical treatment. Gastric aspirate $(10 \mathrm{ml})$ was obtained under sterile conditions and sent for microbiological culture. All septic events were recorded and the microbiology assessed.

A total of 231 patients were studied. Sixty five patients (28\%) had sterile cultures, seventy two $(31 \%)$ grew one organism type and $95(41 \%)$ had multiple organisms cultured from their aspirates. Monoand multiorganism culture were associated with a significant increase in septic complications when compared with no growth (38\% vs $17 \%$ $\left.p<0.05 x^{2}=4.16\right)$ and $\left(56 \%\right.$ vs $\left.17 \%, p<0.001 x^{2}=11.52\right)$ respectively. Coliforms and particularly E. Coli and Strep. Faecaelis were strongly associated with subsequent septic events $(68 \% p<$ $0.001,87 \%$ p $<0.001$ ).

We conclude that colonisation of the upper gastrointestinal tract frequently occurs in ill surgical patients and does predict septic morbidity.
SEQUELAE OF INFECTIOUS DIARRHOEA: HIGH RATE OF GASTRO-INTESTINAL INVESTIGATIONS AND LEVELS OF MORBIDITY. L.M. Hebden', RC. Spiller', K.R Neal' ${ }^{2}$. Depts. of Gastroenterology ${ }^{1}$ and Epidemiology ${ }^{2}$, University of Nottingham Medical School, UK.

The number of cases of notified food poisonings continues to increase. However, little is known about the consequences of food poisoning in terms of patient morbidity and medical care and resource usage.

The statutory notifications system was used to identify 665 people with gastroenteritis over a 6 month period. A questionnaire covering medical care, the episode of gastro-enteritis and sequelae, bowel habit now and 12 months before was mailed 6 months after the notification date. 435 questionnaires (66\%) were retumed. Statistical analyses were done using SPSS for windows.

49 patients $(11 \%)$ were admitted to hospital with no difference in admission rates by age, sex or pathogen. There was 1 death and 1 patient with a perforated colon leading to admission to intensive care. $168(39 \%)$ of the patients had at least 7 days off from work or regular duties. This is an underestimate as very few people above retirement age reported time off work. 90 patients reported weight loss of at least 1 stone with a median time of 8 weeks before regaining the weight, 16 had not regained their original weight at 6 months. Significant weight loss was associated with male sex relative risk $(R R)=1.7(95 \% \mathrm{CI}=1.1-2.6)$, duration of diarthoea $\mathrm{RR}=1.5(95 \% \mathrm{CI}=1.2-1.8)$ for each week of symptoms and vomiting as part of the illness $R R=1.5(95 \% C I=1.1-2.1)$, after controlling for hospital admiscion. 107 patients (25\%) reported a persistent change in bowel habit at 6 months, of which $28(7 \%)$ fitted the Rome criteria for IBS. 21 patients $(5 \%)$ had further investigations including 5 barium enemas, 1 barium meal, 2 OGDs. with the remainder having out-patient assessments.

In conchusion gastro-enteritis results in significant morbidity, time off work and health care resource utilisation.

\section{'Non-steroidals' F272-F277}

F272

MESENTERIC MARGINAL ULCERATION: PREDILICTION FOR CRITICALLY PERFUSED AREAS IN INDOMETHACININDUCED JEJUNAL ULCERS IN THE RAT. A Anthony, C Thrasivoulou, RE Pounder, AJ Wakefield, AP Dhillon Inflammaton Bowel Disease Study Group, Royal Free Hospital School of Medicine, London, UK.

Background/Aim; This study tested the hypothesis that mesenteric marginal ulceration, a feature of both Crohn's disease and indomethacin(indo)-induced villous contraction and subsequent jejunal ulceration in the rat, localises to critically.perfused areas of the gut. Two separate sets of experiments were performed in the rat to $(a)$, correlate the site of indo-induced intestinal injury with the normal vascular anatomy and (b), to compare regional susceptibility of the intestine to injury following either indo or a general insult, that is, ischaemia-reperfusion following mesenteric artery occlusion. Methods; Rats ( $\mathrm{n}=5 /$ group) received either $(a)$ oral indo $10 \mathrm{mg} / \mathrm{kg}$ or vehicle and terminated $4 \mathrm{~h}$ later. The mesenteric artery was perfused with carbon ink and the intestines formalin-fixed. Tissues were cleared with methyl salicylate for visualisation of the vasculature by dissection and light microscopy. (b) Alternatively. anaesthetised rats ( $n=5 /$ group) were treated as follows; bolus indo iv $75 \mathrm{mg} / \mathrm{kg}$ or iv vehicle with histological examination of the intestines $20 \mathrm{~min}$ later; mesenteric artery occlusion $(10 \mathrm{~min})$ then reperfusion (10 min); sham operation $20 \mathrm{~min}$. Results; (a) All indodosed rats, but none of the controls, developed visible focal areas of severe villous contraction at $4 \mathrm{~h}, 99 \%$ of which were localised between vasa recta along the mesenteric margin. Arterial imaging of the control rat small intestines identified a paucity of the submucosal plexus between vasa recta adjacent to jejunal, but not duodenal or ileal, mesenteric margins; in contrast, the jejunal submucosal plexus was extensive on the anti-mesenteric side. (b) Indo $75 \mathrm{mg} / \mathrm{kg}$ iv caused villous contraction of jejunal, but not duodenal or ileal, mesenteric villi between vasa recta. Mesenteric artery ischaemiareperfusion caused shortening of all villi in the jejunum/ ileum. The villi of sham controls were normal. Conclusions; Critically perfused areas of the mesenteric margin of the rat jejunum are susceptible to NSAID injury. Indo-induced villous contraction that precedes jejunal ulceration may arise as a consequence of ischaemia/reperfusion injury to critically perfused areas of the jejunum.

(This study was supported by Glaxo Wellcome, UK). 
REDUCED ACUTE DUODENAL MUCOSAL INJURY IN HUMAN VOLUNTEERS BY NAPROXEN RELEASED BY THE INTESTINAL PROTECTIVE DRUG ABSORPTION SYSTEM D Cullen', FE Murray', N Hudson', D Tierney', CJ Hawkey ${ }^{1}$ 'Division of Gastroenterology, University Hospital, Nottingham NG7 2UH, UK and 'Elan Pharmaceutical Research Corporation, USA.

INTRODUCTION Naprelan is an unique naproxen formulation which uses a proprietary drug delivery technology - Intestinal Protective Drug Absorption System (IPDAS ${ }^{\mathrm{TM}}$ ) which is designed to yield both a rapid and a smooth sustained release component, intended to minimise gastrointestinal mucosal injury.

AIMS To compare acute gastroduodenal mucosal injury associated with Naprelan, Naprosyn and coated aspirin in volunteers.

METHODS Twenty-four volunteers aged 24.8 (range 19-40) years each received on 3 separate occasions 7 days treatment with Naprelan $1000 \mathrm{mg}$ OD, Naprosyn $500 \mathrm{mg}$ BID or aspirin $650 \mathrm{mg}$ QID. Treatment order was randomised by Latin square design and blinding was maintained using a double-dummy technique. Volunteers underwent endoscopy before and after 7 days of each treatment. Mucosal injury was quantified by counting acute mucosal erosions.

RESULTS Twenty-three subjects completed all treatments. After 7 days of Naprelan there were 4.6 (mean) \pm 1.4 (SEM) gastric erosions, compared to $18.3 \pm 2.8(p<0.0001)$ with aspirin and 5.0 $\pm 1.9(p=0.89)$ with Naprosyn. After Naprelan there were $1.8 \pm$ 0.8 duodenal erosions compared to $5.9+1.7(p=0.009)$ with aspirin and $4.6 \pm 1.7(p=0.024)$ Naprosyn. Six small superficial ulcers developed in the duodenal bulb in 4 volunteers on Naprosyn vs 1 on Naprelan and 2 (in 1 volunteer) with aspirin.

CONCLUSIONS In this study Naprelan was well tolerated and the IPDAS ${ }^{\mathrm{TM}}$ drug delivery system may have contributed to reduced duodenal mucosal injury.

This study was designed and conducted by Nottingham GI Trials.

THE INFLUENCE OF AGE, GENDER, SMOKING AND NSAID ON MUCOSAL ADAPTATION TO NSAIDS IN MAN.

GR Lipscomb, WDW Rees. Department of Gastroenterology, Salford Royal Hospitals NHS Trust, Salford.

Introduction: Oral NSAIDs cause acute gastric injury that resolves despite continued administration by a process known as adaptation However little is known about factors that influence this.

Methods: 64 healthy volunteers were given a 28 day course of either etodolac $300 \mathrm{mg}$ bd (13 subjects), naproxen $500 \mathrm{mg}$ bd (24) enteric coated diclofenac (12) or effervescent diclofenac $50 \mathrm{mg}$ bd (17). All subjects were gastroscoped before and 1, 7 and 28 days during drug administration for assessment of gastric mucosal damage using a modified Lanza scale ( $0=$ normal, 4=severe). Subjects were then divided into three catagories: those who adapted completely, those who adapted incompletely and those who showed no adaptation. Subjects falling into these groups were compared with respect to age, gender, smoking and NSAID prescribed using the Pearson Chi-squared test.

Results: Fifty nine subjects (mean age 29.0 years, range 18-70) developed initial gastric injury to NSAIDs of whom $42(70 \%)$ adapted completely, $13(22 \%)$ adapted incompletely and $4(8 \%)$ showed no evidence of adaptation. 8 subjects were aged over 45 years and a greater proportion of these showed no evidence of adaptation ( $25 \%$ vs $4 \%, \chi^{2}$ $=4.86, p=0.03$ ). There was no evidence of gender influencing adaptation. 16 subjects were smokers of whom a greater proportion showed no evidence of adaptation ( $19 \%$ vs $2 \%, \chi^{2}=4.98, p=0.03$ ). A smaller proportion of those who took naproxen (48\%) adapted completely than those who took enteric coated diclofenac (89\%), effervescent diclofenac $(75 \%)$ or etodolac $(91 \%) \quad\left(x^{2}=9.11, p=0.03\right)$

Conclusion: Some adaptation occurred in over $90 \%$ of subjects after 4 weeks but was less frequent in older subjects and smokers. Complete adaptation occurred less frequently with naproxen than other NSAIDs.
GASTRIC MUCOSAL CONTRACTION AND VASCULAR INJURY INDUCED BY INDOMETHACIN FJCEDE NEUTROPHIL INFILTRATION IN THE RAT A Anthony, R Sim, RE Pounder; AJ Wakefield, AP Dhillon. Inflammatory Bowel Disease Study Group. Royal Free Hospital School of Medicine, London, UK.

Background; Indomethacin (indo) causes gastric ulceration by mechanisms that may be neutrophil-independent. Am; To examine the involvement of neutrophils in the earliest gastric microscopic changes induced by indomethacin in the corpus of fasted rats and the antrum of fasted-refed rats. Methods;. Indo po. $30 \mathrm{mg} / \mathrm{kg}$ or vehicle was administered to six groups of fasted rats that were terminated 5 , 15 and $30 \mathrm{~min}$ after dosing. Indo sc. $30 \mathrm{mg} / \mathrm{kg}$ or vehicle was aleo administered to six groups of fasted-refed rats that were terminated 1,2 and $4 \mathrm{~h}$ later. The stomach was arterial perfusion-fixed and examined for visible and microscopic mucosal injury. Sections were haematoxylin-eosin- and reticulin-stained to identify mucosal architectural changes and strined immuno-histochemically for actin, fibrin and neutrophils. Neutrophils were counted within gastric lesions (mean \pm SEM). Results; Indo caused time-dependent corpus and antral injury in fasted and fasted-refed rats respectively. At each site, an early phase of microscopic mucosal injury was idemified that occured prior to neutrophil infiltration. This phase was first identified at $5 \mathrm{~min}$ in the corpus and $1 \mathrm{~h}$ in the antrum; (Neutrophils/lesion in, Fasted rats/corpus lesions $=0 \pm 0$ at 5 mins, $1.6 \pm 0.8$ at $15 \mathrm{~min}$ and $1.1 \pm 0.3$ at $30 \mathrm{~min}$; fasted-refed rats/antral lesion $=0 \pm 0$ at $1 \mathrm{~h}, 0.4 \pm 0.3$ at $2 \mathrm{~h}$ and $49 \pm 22$ at 4 h). All controls showed no neutrophils within equivalent susceptible areas of the stomach. Within the superficial corpus mucosa this phase preceded coagulative necrosis and included surface epithelial expulsion, mucosal contraction with capillary aggregation and distortion, intravascular vascular fibrin deposition and capillary congestion. The antrum showed similar early changes except that full-thickness mucosal coagulative necrosis was an early predominant finding. Conclusion; In two experimental models of NSAID gastric ulceration the mucosa undergoes contraction, vascular fibrin deposition and necrosis prior to neutrophil infiltration. These findings support a neutrophil-independent, ischaemic pathogenesis for NSAID gastric ulceration.

(This study was supported by Glaxo Wellcome, UK).
LOWER FREQUENCY OF GASTRODUODENAL ULCERS AND EROSIONS AND DYSPEPTIC SYMPTOMS IN NSAID USERS DURING MAINTENANCE WITH OMEPRAZOLE COMPARED TO RANITIDINE. CJ Hawkey', AJ Swannell', S Eriksson ${ }^{3}$, A Walan ${ }^{3}, M$ Bjersing $^{3}, M$ Jallinder', G Langstrom ${ }^{3}$, ND Yeomans'. 'Div Gastro, Univ Hospital, ${ }^{2}$ Dept of Rheuma/Rehab, City Hosp, Nottingham UK. ${ }^{3}$ Astra Hassle AB, Sweden. 'Dept of Med, Western Hosp, Melbourne, Australia.

BACKGROUND $\mathrm{H}_{2}$ receptor antagonists are widely used in patients taking non-steroidal anti-inflammatory drugs (NSAIDs), despite evidence that they are of limited efficacy. Acute studies have suggested that omeprazole might be a more effective agent. ADM To compare in a randomised double-blind study the prophylactic effect of omeprazole (ome) $20 \mathrm{mg}$ once daily with that of ranitidine (ran) $150 \mathrm{mg}$ twice daily during 6 months in patients on contimuous NSAID therapy.

METHODS Patients who successfully completed an international healing study and with a need for continuing NSAIDs were re-randomised to omeprazole or ranitidine. Patients were endoscuped after 1,3 and 6 months and in case of suspected relapse or study discontinuation. Study end-points were: ulcer ( $>3 \mathrm{~mm}$ ), more than 10 erosions in stomach or duodenum, more than mild dyspeptic symptoms or discontinuation from treatment due to adverse events (AEs).

RESULTS A total of $\mathbf{4 3 2}$ patients were enrolled into the study and $\mathbf{4 2 5}$ were evaluable for All Patients Treated analysis.

Estimated $\%$ of patients in

remission at 6 months

No end-points reached

No ulcer and $\leq 10$ erosions in stomach/

duodenum and $\leq$ mild dyspeptic symptoms $\} \quad 80 \% \quad 66 \% \quad 0.0003$

Life table analysis showed that significantly more patients were in remission at 6 months on omeprazole compared to ranitidine. The proportion of patients who relapsed with ulcer were $6 \%$ and $20 \%$ for the omeprazole and ranitidine groups, respectively. There was no significant difference between the treatment groups in time to discontinuation due to AEs.

CONCLUSION Omeprazole $20 \mathrm{mg}$ once daily is superior to ranitidine in prevention of gastroduodenal ulcers, erosions and dyspeptic symptoms in NSAID users with a history of NSAID-associated gastroduodenal lesions. This study was funded by Astra Hassle, Sweden. 
REDUCTION IN RELATIVE INCIDENCE OF DUODENAL ULCERS: A LONG-TERM AUDIT.

SGRG Barton. M Newton, CC Hepworth, E Carty, WR Burnham GI Unit, Oldchurch Hospital, Romford.

Introduction: Duorenal ulceration (DU) is a common finding both at routine diagnostic gastroscopy (OGD) and at emergency OGD for bleeding. Following the discovery that Helicobacter pylori (HP) had an important role in the pathogenesis of DU, eradicative therapy became common by 1990 . To determine whether this has effected any change in the incidence of DU, we have now analysed our OGD records for the period 1985-95.

Methods: OGD's performed by the gastroenterology firm are recorded in a computerised database. OGD records for the period 1/1/85-1/12/95 were audited. Yearly records were recorded for: total number of DU's, DU's seen at diagnostic OGD, bleeding DU's and gastric ulcers. These results were expressed as a percentage of the total number of OGD's perfomed in that year.

Results: Expressed as a percentage of the total number of OGD's performed, there were significant reductions in both the total number of DU's $(p<0.02)$ and number of diagnostic DU's $(p<0.02)$ during this ten year period. There were no significant trends seen in the number of gastric ulcers or bleeding DU's during the same period. As expected there was a steady increase in the total number of OGD's performed.

Conclusions: The significant reduction in the percentage of total and diagnostic DU's seen whilst the proportion of gastric ulcers and bleeding DU's has remained constant may reflect (a) increasing treatment of HP during this period and a real reduction in DU prevalence, (b) a lower proportion of OGD's revealing pathology, possibly related to the increasing use of open-access gastroscopy (OAG -1990 onwards). However the proportion of positive findings at OAG has remained constant since 1992 so it seems likely that there has been a real reduction in uncomplicated DU's. The constant proportion of bleeding DU's may reflect NSAID use rather than HP in these patients. 